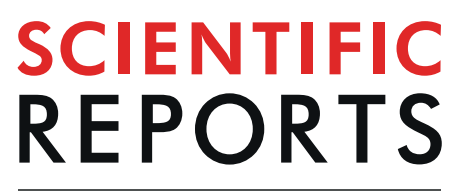

natureresearch

\title{
Clinical Relevance of the serum CTLA-4 in Cats with Mammary Carcinoma
}

\author{
Ana Catarina Urbano ${ }^{1}$, Catarina Nascimento ${ }^{1}$, Maria Soares ${ }^{2}$, Jorge Correia ${ }^{1} \&$ \\ Fernando Ferreira $^{1 *}$
}

Cytotoxic T lymphocyte associated antigen 4 (CTLA-4) serves an important role in breast cancer progression, which has led to the development of novel immunotherapies aimed at blocking tumor immune evasion. Although feline mammary carcinoma is increasingly recognized as a valuable cancer model, no studies on CTLA-4 function had been conducted in this species. The serum CTLA-4, TNF- $\alpha$ and IL- 6 levels of 57 female cats with mammary carcinoma were determined by ELISA, and immunohistochemistry was performed to evaluate CTLA-4 and FoxP3 expression in tumor cells and interstitial lymphocytes. The results obtained show that serum CTLA-4 levels are increased in cats with mammary carcinoma $(P=0.022)$, showing an association with a number of clinicopathological features: smaller tumor size, $P<0.001$; absence of tumor necrosis, $P<0.001$; non-basal status, $P<0.02$ and HER-2-positive status. Additionally, a strong positive correlation was found between serum CTLA-4 levels and serum TNF- $\alpha(R=0.88, P<0.001)$ and IL- 6 levels $(R=0.72, P<0.001)$. Concerning the CTLA-4 and FoxP3 expression, although detected in both interstitial lymphocytes and tumor cells, a positive association was found only between interstitial CTLA- 4 and FoxP3 expressions $(R=0.387$, $P=0.01)$, which is negatively associated with the serum CTLA-4 levels $(P=0.03)$. These findings provide a preliminary step in the characterization of immune profiles in feline mammary carcinoma, uncovering a molecular rationale for targeted therapy with CTLA-4 pathway inhibitors. Finally, by strengthening the hypothesis of an immunomodulatory role for this regulator, we further validate the utility of spontaneous feline mammary carcinoma as a model for human breast cancer.

Although the dog has been the focus in comparative oncology, cats also have clear benefits over rodent laboratory models of cancer, namely, they are immunocompetent and share the same environment as humans, reflecting more accurately the complex interplay between genetics and environmental risk factors, as well as the role of the immune system and tumor microenvironment (TME). Furthermore, the higher similarity between the cat and human genomes, together with the increased frequency of several tumor types (e.g. injection-site sarcoma, oral squamous cell carcinoma, lymphoma and malignant mammary tumors) ${ }^{1-3}$, anticipate that the cat may be a superior model. Feline mammary carcinoma shares many epidemiological and histopathological characteristics with human breast cancer, in particular, the human epidermal receptor-2 (HER-2) positive and triple negative (TN) subtypes, having been proposed as a suitable model for their study ${ }^{4-6}$, and offers further opportunities for studying certain aspects of tumor biology, such as the crosstalk between the immune system and tumor development.

Chronic inflammation is a well-established risk factor for several cancers, with lymphocytes playing a pivotal role in the development of chronic inflammatory conditions ${ }^{7}$. The activation of T-lymphocytes requires two signals: after recognition of the MHC-peptide complex by the T-lymphocyte receptor (TCR), a second signal is provided by the binding of the cluster of differentiation 28 (CD28) to its ligands CD80/CD86 on antigen presenting cells (APCs). This interaction leads to Cytotoxic T-lymphocyte associated protein 4 (CTLA-4, CD152) translocation to the cell surface. CTLA- 4 is an immunoglobulin superfamily cell adhesion molecule, exclusively expressed on lymphocytes, and a homologue of CD28. Because CTLA-4 has higher affinity for CD80/CD86 it can interrupt the activation signal delivered by CD28 and deliver its own signal which downregulates T-lymphocyte

${ }^{1}$ CIISA - Centro de Investigação Interdisciplinar em Sanidade Animal, Faculdade de Medicina Veterinária, Universidade de Lisboa, Avenida da Universidade Técnica, Lisboa, 1300-477, Portugal. ${ }^{2}$ Research Center for Biosciences and Health Technologies (CBiOS), Faculdade de Medicina Veterinária, Universidade Lusófona de Humanidades e Tecnologias (ULHT), Lisboa, 1749-024, Portugal. *email: fernandof@fmv.ulisboa.pt 
a

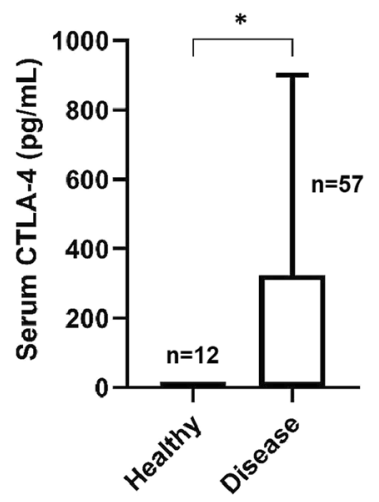

d

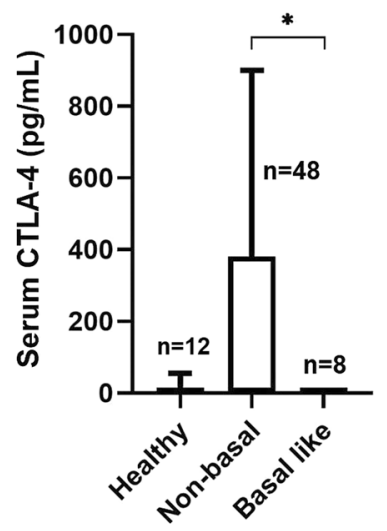

b

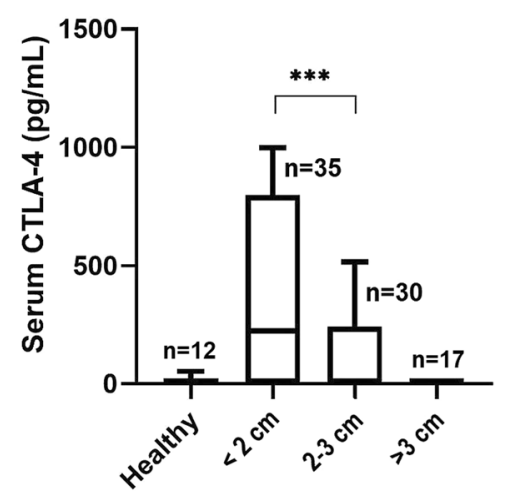

e

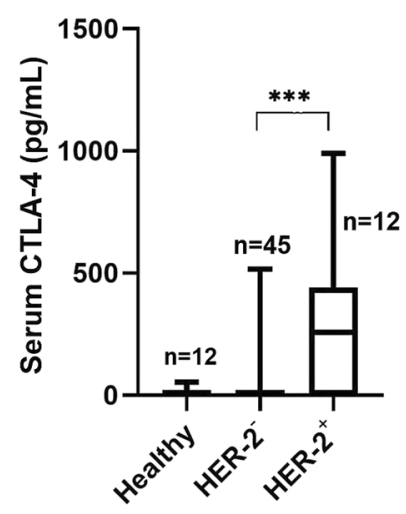

C

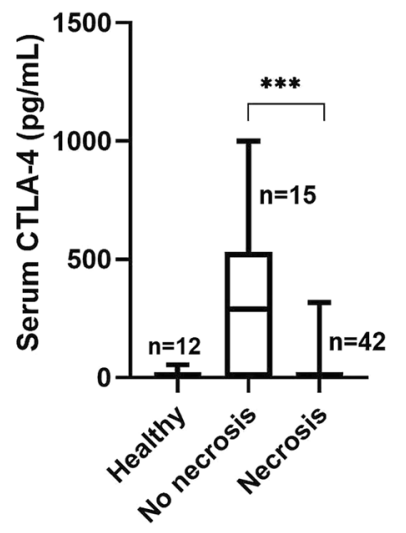

Figure 1. Box plot analysis of serum cytotoxic T-lymphocyte associated protein 4 (CTLA-4) levels in cats with mammary carcinoma and their association with clinicopathological and immunohistochemical parameters: (a) disease status (b) tumor size (cm); (c) tumor necrosis; (d) basal status; (e) HER-2 status. $* P<0.05$; $* * P<0.001$; $* * * P<0.0001$.

function ${ }^{8}$, triggering a negative feedback loop that is essential to the maintenance of immune self-tolerance and homeostasis. Feline CTLA-4 shows a high homology with the human and murine counterparts ( $86.6 \%$ and $76.2 \%$ respectively), presenting an hexapeptide motif (MYPPPY) within the extracellular domain responsible for interaction with the B7 ligands that is highly conserved among mammals?

In humans, CTLA-4 also exists as a soluble isoform (sCTLA-4) in sera, an alternative splicing product of the CTLA-4 gene, mainly expressed on resting T-lymphocytes cells or at the post-activated state ${ }^{10}$. Elevated sCTLA-4 levels have been reported in several cancers, including esophageal ${ }^{11}$, lung ${ }^{12}$, and breast carcinoma ${ }^{13}$, however, the clinical implications of CTLA-4 in the tumor microenvironment are still controversial. While it has been demonstrated that CTLA- 4 expression can be an important mechanism of tumor immune evasion ${ }^{14,15}$, with high levels of CTLA-4 expression in tumor tissues being associated with poor overall survival ${ }^{16}$, a recent study on breast cancer patients found that elevated sCTLA-4 levels were associated with improved survival ${ }^{17}$ and several other studies have showed significant correlations between CTLA- 4 and improved OS in other cancer types ${ }^{12}$.

Since sCTLA-4 has also been found to be elevated in several inflammatory human disorders, where it's able to display functional activities, acting on the proliferation capability of T-lymphocytes and modulating the secretion of cytokines ${ }^{18}$, we hypothesized that the elevated levels of sCTLA- 4 in breast cancer might be a reflection of crosstalk between immune cells and the TME. Our investigation, therefore, studied the relationship between serum CTLA-4 and standard inflammatory markers, namely the pro-inflammatory cytokines, TNF- $\alpha$ and IL- 6 , which have emerged as central players linking inflammation and cancer. To further investigate this crosstalk, we evaluated the expression of CTLA-4 and FoxP3, an immune cell marker for regulatory T-lymphocytes (Tregs), in the TME. Tregs are a subset of CD4+ T-lymphocytes, known to highly infiltrate various tumor types in both humans and felines ${ }^{19}$, that, unlike other T-lymphocyte subsets, express CTLA- 4 constitutively, and seem to be the primary source of sCTLA- $4^{20}$.

Results

Cats with mammary carcinoma show increased serum CTLA-4 levels being related with small tumor size, absence of tumor necrosis, non-basal status and HER-2 positive status. Serum CTLA-4 levels were detected in 23 of the 54 cats with mammary carcinoma (43\%), showing a median of $459.4 \mathrm{pg} /$ $\mathrm{mL}$ (range 77-999.3 pg/mL), contrasting with undetectable levels in all healthy animals (detection limit $=31.3 \mathrm{pg} /$ $\mathrm{mL}$ ). As such, serum CTLA-4 levels in the cancer group were significantly higher than those in the healthy group 
a

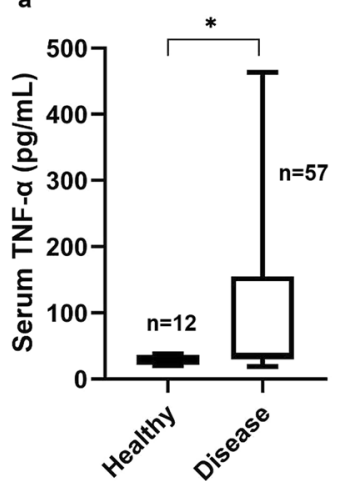

d

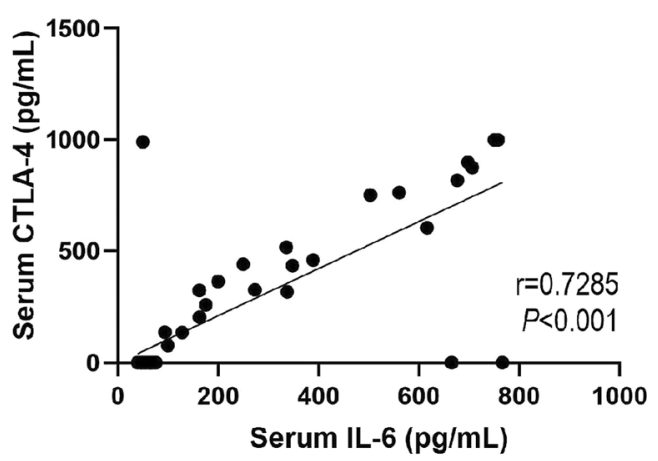

b

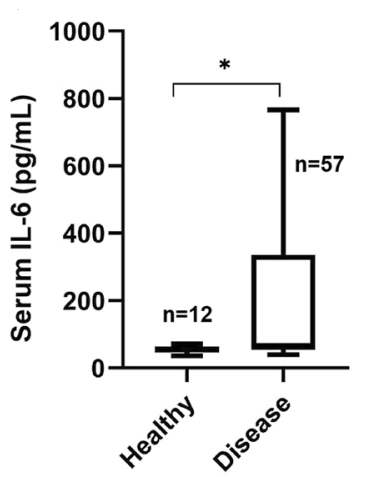

C

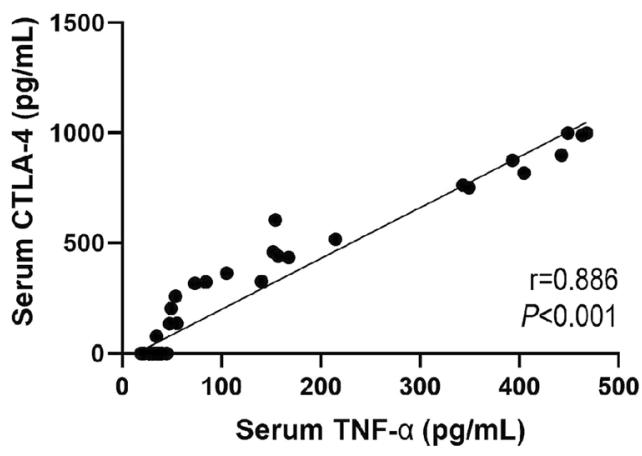

e

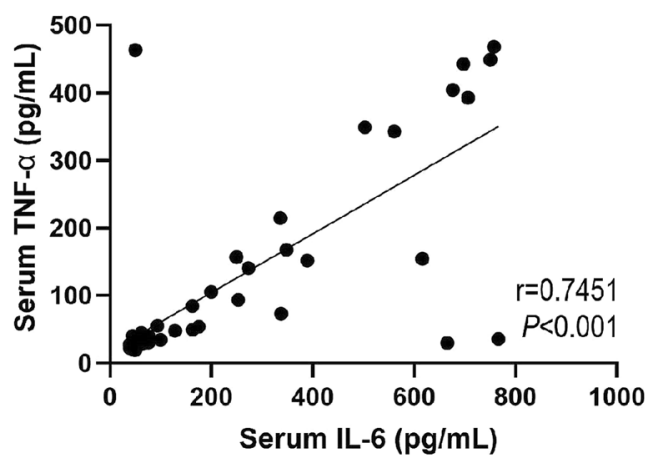

Figure 2. Box plot analysis of serum cytokine levels and Pearson correlation with serum cytotoxic T-lymphocyte associated protein 4 (CTLA-4): (a) tumor necrosis factor alpha (TNF- $\alpha$ ) serum levels; (b) Interleukin 6 (IL-6) serum levels; (c) correlation between serum CTLA-4 and TNF- $\alpha(\mathrm{R}=0.8860, \mathrm{P}<0.001)$; (d) correlation between serum CTLA- 4 and IL- $6(\mathrm{R}=0.7285, \mathrm{P}<0.001)$; (e) correlation between TNF- $\alpha$ and IL-6 $(\mathrm{R}=0.7451, \mathrm{P}<0.001) . * P<0.05 ; * * P<0.001 ; * * * P<0.0001$.

$(P=0.022$; Fig. 1a). Further statistical analysis showed that higher serum CTLA-4 levels are associated with small tumor size $(P<0.001$; Fig. 1b), absence of tumor necrosis $(P<0.001$; Fig. 1 c), and non-basal status $(P=0.002$; Fig. 1d). Serum CTLA-4 levels were also significantly increased in cats with mammary carcinomas immunohistochemically classified as HER-2-positive $(P<0.001$; Fig. 1e).

Serum CTLA-4 levels are correlated with serum TNF- $\alpha$ and IL- 6 levels. Serum TNF- $\alpha$ and IL- 6 levels were significantly higher in cats with mammary carcinoma compared to healthy controls $(P=0.011$ and $P=0.021$ respectively; Fig. 2a,b), being detectable in all cats with mammary carcinoma enrolled in the study, showing a median of $36.10 \mathrm{pg} / \mathrm{mL}$ (range $19.11-463.5 \mathrm{pg} / \mathrm{mL}$ ) and $65.94 \mathrm{pg} / \mathrm{mL}$ (range 39.17-766.5 pg/ $\mathrm{mL}$ ), respectively. As previously reported, cytokine levels were also detectable in healthy animals, showing a median of $32.79 \mathrm{pg} / \mathrm{mL}$ (range $20.36-37.5 \mathrm{pg} / \mathrm{mL}$ ) and $56.48 \mathrm{pg} / \mathrm{mL}$ (range $36.09-71.07 \mathrm{pg} / \mathrm{mL}$ ), respectively. In addition, a strong positive correlation was found between serum CTLA- 4 and both serum TNF- $\alpha(R=0.8860$, $P<0.001$; Fig. $2 c)$ and IL-6 levels $(R=0.7285, P<0.001$; Fig. $2 \mathrm{~d})$, and also between serum TNF- $\alpha$ and IL-6 levels $(R=0.7451, P<0.001$; Fig. 2e).

CTLA-4 is expressed in interstitial lymphocytes being negatively correlated with serum CTLA-4 levels in cats with mammary carcinoma. Immunostaining analysis revealed that from the 49 tumor specimens evaluated, 34 (81\%) showed a CTLA-4 positive expression in interstitial lymphocytes and $2(4 \%)$ in tumor cells (Table 1). While in interstitial lymphocytes, CTLA-4 expression was detected in the cell membrane and cytoplasm, in tumor cells, the staining was exclusively cytoplasmic. Both interstitial lymphocytes and tumor cells showed a granular staining pattern of differing intensities (Fig. 3). A significant negative correlation was found between serum CTLA-4 levels and CTLA-4 expression in interstitial lymphocytes $(R=-0.3425, P=0.03)$ (Fig. 4a). To further confirm this association, tumors were divided into CTLA-4-positive and CTLA-4-negative groups, according to the expression of CTLA-4 in interstitial lymphocytes. The strong negative association between the serum levels and interstitial CTLA-4 expression was reconfirmed by the Mann-Whitney U test $(P=0.03)$ (Fig. 4b). No CTLA-4 expression was detected in the normal control tissue (data not shown).

FoxP3 and CTLA-4 expression is correlated in interstitial lymphocytes. From the 49 tumor specimens, 35 (74\%) and 15 (31\%) were scored as FoxP3-positive in interstitial lymphocytes and in tumor cells, respectively (Table 2). Whereas in interstitial lymphocytes, most of FoxP3 staining was detected in the cytoplasm with some cells exhibiting nuclear expression (Fig. 3e), only discrete FoxP3 ${ }^{+}$dots were found in the nucleus of 


\begin{tabular}{|c|c|c|c|c|c|}
\hline No & +Cells (\%) & Intensity & Score & Interpretation & $+\mathrm{TC}(\%)$ \\
\hline 1 & 0 & 0 & 0 & Negative & 0 \\
\hline 2 & 5 & $2+$ & 2 & Positive & 0 \\
\hline 3 & 2 & $2+$ & 2 & Positive & 0 \\
\hline 4 & 5 & $3+$ & 3 & Positive & 0 \\
\hline 5 & 0 & 0 & 0 & Negative & 0 \\
\hline 6 & 10 & $3+$ & 6 & Positive & 0 \\
\hline 7 & 5 & $3+$ & 3 & Positive & 0 \\
\hline 8 & 0 & 0 & 0 & Negative & 0 \\
\hline 9 & 5 & $3+$ & 3 & Positive & 0 \\
\hline 10 & 5 & $3+$ & 3 & Positive & 0 \\
\hline 11 & 1 & $2+$ & 2 & Positive & 0 \\
\hline 12 & 15 & $2+$ & 4 & Positive & 0 \\
\hline 13 & 5 & $2+$ & 2 & Positive & 0 \\
\hline 14 & 0 & 0 & 0 & Negative & 0 \\
\hline 15 & 2 & $2+$ & 2 & Positive & 0 \\
\hline 16 & NS & NS & ND & ND & 0 \\
\hline 17 & NS & NS & ND & ND & 0 \\
\hline 18 & NS & NS & ND & ND & 0 \\
\hline 19 & NS & NS & ND & ND & 0 \\
\hline 20 & 2 & $2+$ & 2 & Positive & 0 \\
\hline 21 & 3 & $1+$ & 1 & Negative & 0 \\
\hline 22 & 3 & $3+$ & 3 & Positive & 0 \\
\hline 23 & NS & NS & ND & ND & 0 \\
\hline 24 & 2 & $2+$ & 2 & Positive & 0 \\
\hline 25 & 1 & $1+$ & 1 & Negative & 0 \\
\hline 26 & 20 & $2+$ & 4 & Positive & 0 \\
\hline 27 & 30 & $3+$ & 6 & Positive & 0 \\
\hline 28 & 20 & $3+$ & 6 & Positive & 0 \\
\hline 29 & 10 & $1+$ & 2 & Positive & 0 \\
\hline 30 & NS & NS & ND & ND & 0 \\
\hline 31 & 1 & $1+$ & 1 & \begin{tabular}{|l|} 
Negative \\
\end{tabular} & 0 \\
\hline 32 & 20 & $2+$ & 4 & Positive & 1 \\
\hline 33 & 1 & $1+$ & 1 & Negative & 0 \\
\hline 34 & 20 & $3+$ & 6 & Positive & 0 \\
\hline 35 & 40 & $2+$ & 6 & Positive & 0 \\
\hline 36 & 30 & $3+$ & 6 & Positive & 0 \\
\hline 37 & 1 & $2+$ & 2 & Positive & 0 \\
\hline 38 & 2 & $2+$ & 2 & Positive & 0 \\
\hline 39 & 2 & $2+$ & 2 & Positive & 0 \\
\hline 40 & 40 & $3+$ & 9 & Positive & 0 \\
\hline 41 & NS & NS & ND & ND & 0 \\
\hline 42 & 50 & $2+$ & 6 & Positive & 0 \\
\hline 43 & 30 & $3+$ & 6 & Positive & 3 \\
\hline 44 & 15 & $2+$ & 4 & Positive & 0 \\
\hline 45 & 10 & $2+$ & 4 & Positive & 0 \\
\hline 46 & 40 & $3+$ & 9 & Positive & 0 \\
\hline 47 & 10 & $3+$ & 6 & Positive & 0 \\
\hline 48 & 3 & $3+$ & 3 & Positive & 0 \\
\hline 49 & 5 & $2+$ & 2 & Positive & 0 \\
\hline
\end{tabular}

Table 1. Evaluation of CTLA-4 positivity in interstitial lymphocytes showing percentages of positive cells (+Cells), intensity of the staining reactions, IHC scores, interpretation, and percentages of stained tumor cells (TC). NS - none seen; ND - not determined.

tumor cells (Fig. 3f). Although, no significant correlation was observed between serum CTLA-4 levels and FoxP3 expression in either interstitial lymphocytes or tumor cells, FoxP3 expression in interstitial lymphocytes was strongly correlated with the CTLA- 4 IHC score of interstitial lymphocytes $(R=0.387, P=0.01$, Fig. $4 \mathrm{c})$. No FoxP3 expression was detected in the normal control tissue (data not shown). 


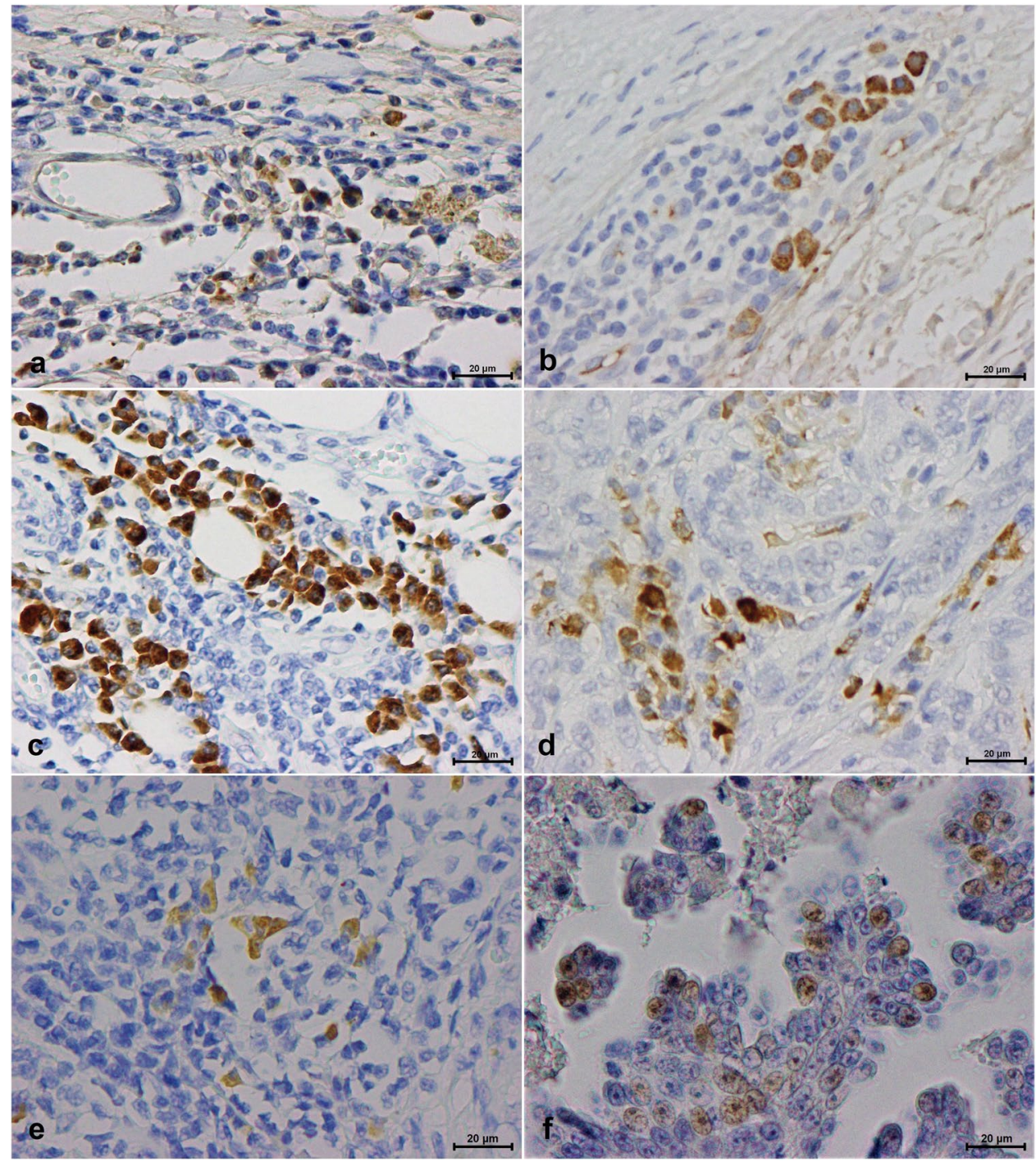

Figure 3. Serum cytotoxic T-lymphocyte associated protein 4 (CTLA-4) and Forkhead box (Fox)P3 expression in interstitial lymphocytes and tumor tissue: (a) CTLA-4 intensity $1+$, showing a low number of reactive cytoplasmic granules (from \#21; Table 1); (b) CTLA-4 intensity $2+$, showing a moderate number of reactive cytoplasmic granules (from \#26; Table 1); (c) CTLA-4 intensity 3+, showing a high number of reactive granules packed in the cytoplasm (from \#28; Table 1); (d) CTLA-4 expression in tumor tissue; (e) FoxP3 interstitial expression; (f) FoxP3 expression in tumor tissue (40x objective).

\section{Discussion}

Cats are increasingly being used as models for the study of spontaneous human diseases, and the epidemiological and histological similarities between feline mammary tumors and human breast cancer make this species a valuable cancer model for comparative oncology studies. To the best of our knowledge, the present study was the first to evaluate the serum CTLA-4 profiles and the serum TNF- $\alpha$ and IL- 6 levels in cats with mammary carcinoma. The results presented clearly demonstrate that serum CTLA-4 levels are increased in cats with mammary carcinoma, in accordance with previous results reported in human malignant tumors ${ }^{11-13}$. This event may relate to an ongoing inflammatory response in the tumor microenvironment, where post-activated T-lymphocytes are actively expressing sCTLA-4. In parallel, the proteolytic cleavage of the full length isoform (mCTLA-4) concurrent with T-lymphocyte exhaustion, a progressive loss of effector function due to prolonged antigen stimulation observed in cancer patients, may also be contributing factors ${ }^{21}$. Results obtained also demonstrated that higher serum CTLA-4 levels are correlated with a number of clinicopathological features (i.e. smaller tumors, absence of necrosis, HER2-positive status, and non-basal status). Although these findings seem to be counterintuitive as increasing concentrations of CTLA-4 should inhibit T-lymphocyte activity, some studies have suggested that, while in the resting T-lymphocytes only sCTLA-4 is expressed, inhibiting CD28-ligand interactions, in later tumor phases, mCTLA- 4 is overexpressed, with sCTLA-4 interfering with mCTLA-4-ligand interactions, thus enhancing T-lymphocyte reactivity by preventing the transduction of inhibitory signals ${ }^{18,22,23}$. It has also been suggested that CTLA-4 can mediate negative signals into tumor cells, comparable to those observed in resting 

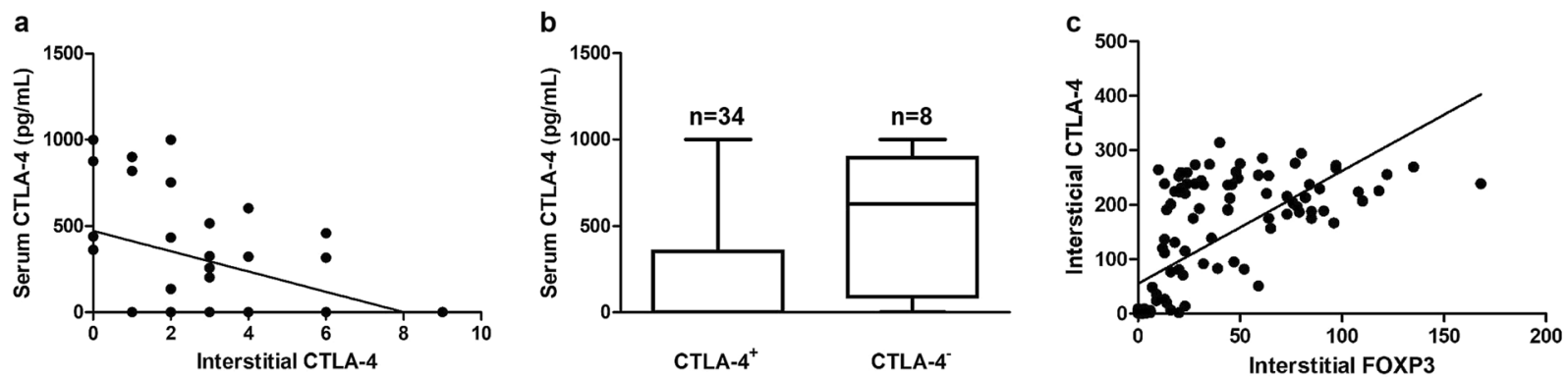

Figure 4. Associations of serum cytotoxic T-lymphocyte associated protein 4 (CTLA-4) levels with interstitial CTLA-4 and FoxP3 expression: (a) Spearman rank test of CTLA-4 serum levels and CTLA-4 interstitial expression; (b) Mann-Whitney U test of CTLA-4 serum levels and CTLA-4 interstitial expression; (c) Pearson correlation of CTLA-4 serum levels and FoxP3 interstitial expression.

T-lymphocytes, thus inhibiting tumor cell proliferation and/or inducing apoptotic cell death ${ }^{24}$. In this context, the association found between elevated serum CTLA-4 and HER2-positive tumors would seem paradoxical, as this subtype is frequently associated with more aggressive features and poorer prognosis in humans. However, a recent study reported a positive correlation between higher HER2 mRNA levels and better clinical outcome in feline mammary carcinoma ${ }^{25}$, while a study using a mouse model of HER2 positive breast cancer found that induced intratumoral expression of a CTLA-4 monoclonal antibody not only failed to exert anti-tumor effects, but instead stimulated tumor growth ${ }^{26}$, in support of our results.

In human breast cancer most cytokines are overexpressed, with patients showing increased serum levels ${ }^{27}$. Accordingly, our findings revealed an association between cats with mammary carcinoma and higher serum levels of two pro-inflammatory cytokines (TNF- $\alpha$ and IL-6), positively correlated with serum CTLA-4 levels, as reported in humans and mice ${ }^{21,28}$. A possible reason for this association may be the activation of certain cell types involved in cancer-associated inflammation, like Tregs. Indeed, our results revealed that most tumor samples scored as FoxP3-positive in interstitial lymphocytes (74\%), suggesting Treg tumor infiltration, and strongly correlated with interstitial CTLA-4 expression. Interestingly, a significant proportion of the tumors also scored as FoxP3-positive (31\%), showing a nuclear staining pattern, in accordance with that described for human breast cancers $^{29}$.

Finally, our results revealed CTLA-4 expression in the cell membrane and cytoplasm of interstitial lymphocytes, showing a strong negative correlation with serum CTLA-4 levels. This relation might indicate the presence of memory T-lymphocytes in more advanced disease stages, as breast cancer patients are known to have significantly more circulating memory T-lymphocytes and fewer naïve T-lymphocytes, and tumor progression and aggressiveness seem to favor the expansion of the former over the latter ${ }^{30}$. Intracellular stores of CTLA-4 have been shown to be very low in naïve T-lymphocytes, whilst significant amounts are present in memory T-lymphocytes ${ }^{31}$. Although, the overexpression of CTLA-4 in interstitial lymphocytes has been reported in human breast cancer before ${ }^{32}$, it's correlation with serum CTLA-4 levels, to our best knowledge, had never been studied.

In conclusion, the present study demonstrated a positive association between serum CTLA-4 levels and two pro-inflammatory cytokine serum levels in cats with mammary carcinoma, with higher serum CTLA-4 levels being associated with less aggressive clinicopathological features. We further identified expression of CTLA-4 and FoxP3 in both interstitial and tumor cells, with a positive association between interstitial expression of CTLA-4 and FoxP3, which was also associated with lower serum CTLA-4 levels. Future investigations including a larger and more diverse patient cohort are required to confirm the results, and evaluation of additional T-lymphocyte immune markers and activation phenotypes of various immune cells (including PD-1 and PD-L1) is required to more fully define immune profiles of feline mammary carcinoma patients. Nevertheless, our findings provide further support for spontaneous feline mammary carcinoma as a model for human breast cancer, that can be used to investigate novel immunotherapies that may benefit both human and feline patients.

\section{Materials and Methods}

Study population. All animals enrolled in this study had a fully documented history of feline mammary carcinoma and were followed up at the Faculty of Veterinary Medicine Teaching Hospital (HEV) between June 2011, and September 2013. Available historical data included age, clinical stage (TNM), malignancy grade, tumor burden and size, regional lymph node involvement, presence of tumor necrosis, lymphatic vessel invasion, lymphocyte infiltration or cutaneous ulceration and histopathological classification (ER status, PR status, HER-2 status, basal status, Ki67 index) (Table 3). Serum samples from 57 female cats were collected at time of admission and conducted after the written consent of the pet owner, in accordance with the principles and procedures outlined in the NIH Guide for the Care and Use of Animals, aliquoted and stored at $-80^{\circ} \mathrm{C}$. For 49 of these animals, matching formalin-fixed and paraffin-embedded tumor tissue sections were evaluated. In parallel, serum samples from twelve healthy cats were used as controls for the quantification of serum CTLA-4, TNF- $\alpha$ and IL-6 levels.

Measurement of CTLA-4 and cytokine serum levels. Serum samples were kept frozen at $-80^{\circ} \mathrm{C}$ and thawed shortly before determination of CTLA-4, TNF- $\alpha$ and IL- 6 serum levels. Commercially available 


\begin{tabular}{|c|c|c|c|c|c|}
\hline No & +Cells (\%) & Intensity & Score & Interpretation & $+\mathrm{TC}(\%)$ \\
\hline 1 & 5 & $2+$ & 2 & Positive & 0 \\
\hline 2 & 1 & $2+$ & 2 & Positive & 1 \\
\hline 3 & 5 & $3+$ & 3 & Positive & 0 \\
\hline 4 & 30 & $3+$ & 6 & Positive & 0 \\
\hline 5 & 1 & $2+$ & 2 & Positive & 1 \\
\hline 6 & 1 & $2+$ & 2 & Positive & 0 \\
\hline 7 & 3 & $3+$ & 3 & Positive & 0 \\
\hline 8 & 1 & $2+$ & 2 & Positive & 1 \\
\hline 9 & 1 & $2+$ & 2 & Negative & 3 \\
\hline 10 & 4 & $3+$ & 3 & Positive & 0 \\
\hline 11 & 2 & $2+$ & 2 & Positive & 0 \\
\hline 12 & 20 & $2+$ & 4 & Positive & 0 \\
\hline 13 & 5 & $2+$ & 2 & Positive & 3 \\
\hline 14 & 2 & $1+$ & 1 & Negative & 0 \\
\hline 15 & 3 & $3+$ & 3 & Positive & 0 \\
\hline 16 & 5 & $3+$ & 3 & Positive & 3 \\
\hline 17 & 1 & $3+$ & 3 & Positive & 0 \\
\hline 18 & 10 & $3+$ & 6 & Positive & 0 \\
\hline 19 & 1 & $2+$ & 2 & Positive & 5 \\
\hline 20 & 1 & $2+$ & 2 & Positive & 0 \\
\hline 21 & 10 & $1+$ & 2 & Positive & 10 \\
\hline 22 & 10 & $2+$ & 4 & Positive & 10 \\
\hline 23 & 0 & 0 & 0 & Negative & 0 \\
\hline 24 & 30 & $2+$ & 4 & Positive & 1 \\
\hline 25 & 1 & $1+$ & 2 & Positive & 0 \\
\hline 26 & 20 & $2+$ & 4 & Positive & 0 \\
\hline 27 & 20 & $2+$ & 4 & Positive & 0 \\
\hline 28 & 10 & $3+$ & 6 & Positive & 1 \\
\hline 29 & 1 & $1+$ & 1 & Negative & 1 \\
\hline 30 & 5 & $2+$ & 2 & Positive & 1 \\
\hline 31 & 1 & $1+$ & 1 & \begin{tabular}{|l|} 
Negative \\
\end{tabular} & 0 \\
\hline 32 & 1 & $2+$ & 2 & Positive & 0 \\
\hline 33 & 0 & 0 & 0 & Negative & 0 \\
\hline 34 & 10 & $3+$ & 6 & Positive & 0 \\
\hline 35 & 0 & 0 & 0 & Negative & 0 \\
\hline 36 & 15 & $3+$ & 6 & Positive & 1 \\
\hline 37 & 0 & 0 & 0 & Negative & 0 \\
\hline 38 & 1 & $2+$ & 2 & Positive & 0 \\
\hline 39 & 0 & 0 & 0 & Negative & 0 \\
\hline 40 & 0 & 0 & 0 & Negative & 1 \\
\hline 41 & 0 & 0 & 0 & Negative & 0 \\
\hline 42 & 3 & $2+$ & 2 & Positive & 0 \\
\hline 43 & 3 & $3+$ & 3 & Positive & 0 \\
\hline 44 & NS & NS & ND & ND & 0 \\
\hline 45 & 1 & $2+$ & 2 & Positive & 0 \\
\hline 46 & 2 & $3+$ & 3 & Positive & 0 \\
\hline 47 & 1 & $3+$ & 3 & Positive & 0 \\
\hline 48 & 0 & 0 & 0 & \begin{tabular}{|l|} 
Negative \\
\end{tabular} & 0 \\
\hline 49 & NS & NS & ND & ND & 0 \\
\hline
\end{tabular}

Table 2. Evaluation of FoxP3 positivity in interstitial lymphocytes showing the percentages of positive cells (+Cells), intensity of the staining reactions, IHC scores, interpretation, and percentages of stained tumor cells (TC). NS - none seen; ND - not determined.

immunoassay kits from R\&D Systems (R\&D Systems, Minneapolis, MN, USA) were used according to the manufacturers' instructions. CTLA-4 levels were determined with the Mouse CTLA-4 DuoSet ELISA immunoassay kit (code DY476); TNF- $\alpha$ levels were determined with the Feline TNF- $\alpha$ DuoSet ELISA immunoassay kit (code DY2586); and IL-6 levels were determined with the Feline IL-6 DuoSet ELISA immunoassay kit (code DY2305). 


\begin{tabular}{|c|c|c|}
\hline \multicolumn{2}{|c|}{ Clinical feature } & \multirow{2}{*}{\begin{tabular}{|l|} 
Number (\%) \\
$40(70)$
\end{tabular}} \\
\hline \multirow{5}{*}{ Breed } & Crossbred & \\
\hline & Persian & $7(12)$ \\
\hline & Siamese & $7(12)$ \\
\hline & NFC & $2(4)$ \\
\hline & Russian blue & $1(2)$ \\
\hline \multirow{3}{*}{ Age (years) } & $<8$ & $4(7)$ \\
\hline & $8-12$ & $31(54)$ \\
\hline & $>12$ & $22(39)$ \\
\hline \multirow{4}{*}{ Stage } & I & $15(26)$ \\
\hline & II & $7(12)$ \\
\hline & III & $31(54)$ \\
\hline & IV & $4(7)$ \\
\hline \multirow{3}{*}{ Grade } & 1 & $3(5)$ \\
\hline & 2 & $8(14)$ \\
\hline & 3 & $46(81)$ \\
\hline \multirow{2}{*}{ Burden } & Single tumor & $21(37)$ \\
\hline & Multiple tumors & $36(63)$ \\
\hline \multirow{3}{*}{ Size $(\mathrm{cm})$} & $<2$ & $20(35)$ \\
\hline & $2-3$ & $20(35)$ \\
\hline & $>3$ & $17(30)$ \\
\hline \multirow{3}{*}{ LN status } & Negative & $35(62)$ \\
\hline & Positive & $18(32)$ \\
\hline & ND & $4(7)$ \\
\hline \multirow{2}{*}{ LVI } & No LVI & $50(88)$ \\
\hline & LVI & $7(12)$ \\
\hline \multirow{3}{*}{ LI } & No LI & $16(28)$ \\
\hline & LI & $39(68)$ \\
\hline & ND & $2(4)$ \\
\hline \multirow{2}{*}{ Necrosis } & No necrosis & $15(26)$ \\
\hline & Necrosis & $42(74)$ \\
\hline \multirow{2}{*}{ Ulceration } & No ulceration & $50(88)$ \\
\hline & Ulceration & $7(12)$ \\
\hline \multirow{3}{*}{ Ki67 index } & Low $(<14 \%)$ & $18(32)$ \\
\hline & High $(>14 \%)$ & $38(66)$ \\
\hline & ND & $1(2)$ \\
\hline \multirow{2}{*}{ ER status } & Negative & $39(68)$ \\
\hline & Positive & $18(32)$ \\
\hline \multirow{2}{*}{ PR status } & Negative & $30(53)$ \\
\hline & Positive & $27(47)$ \\
\hline \multirow{2}{*}{ HER-2 status } & Negative & $45(79)$ \\
\hline & Positive & $12(21)$ \\
\hline \multirow{2}{*}{ TN status } & Non-TN & $42(74)$ \\
\hline & $\mathrm{TN}$ & $15(26)$ \\
\hline \multirow{3}{*}{ Basal status } & Non-basal & $48(84)$ \\
\hline & Basal like & $8(14)$ \\
\hline & ND & $1(2)$ \\
\hline
\end{tabular}

Table 3. Clinicopathological characteristics of female cats with mammary carcinoma enrolled in the study $(\mathrm{n}=57)$. LN - Lymph Node; LVI - Lymphatic Vessel Invasion; LI - Lymphocyte Infiltration; ER - Estrogen Receptor; PR - Progesterone Receptor; HER - Epidermal Growth Factor Receptor; TN - Triple Negative; ND Not Determined.

All kit components were stored at $4{ }^{\circ} \mathrm{C}$. A seven-point standard curve was prepared for each assay by making serial dilutions from a stock of recombinant mouse CTLA-4, feline TNF- $\alpha$ and feline IL- 6 provided in the kits. The immunoassays used a solid-phase sandwich enzyme-linked immunosorbent assay (ELISA) technique. Briefly, 96-well microplates were prepared by adding Capture Antibody to each well and incubated overnight. To prevent nonspecific binding the plates were treated with $1 \%$ BSA in PBS for $1 \mathrm{~h}$. Afterwards, diluted serum samples were added to each well and incubated for $2 \mathrm{~h}$. Then, the plates were incubated with Biotinylated Detection Antibody 


\begin{tabular}{|l|l|l|}
\hline Antigen & $\begin{array}{l}\text { Source clone } \\
\text { (code) }\end{array}$ & Dilution \\
\hline $\begin{array}{l}\text { Synthetic peptide corresponding to } \\
\text { human CTLA-4 (internal sequence) }\end{array}$ & $\begin{array}{l}\text { Rabbit monoclonal } \\
\text { (Abcam SP355) }\end{array}$ & $1: 100\left(30^{\prime}\right)$ \\
\hline FoxP3 fusion protein & $\begin{array}{l}\text { Mouse monoclonal } \\
\text { (Abcam 236A/E7) }\end{array}$ & $1: 100\left(30^{\prime}\right)$ \\
\hline
\end{tabular}

Table 4. Primary antibodies and their conditions of use.

for $2 \mathrm{~h}$ and Streptavidin Conjugated to Horseradish Peroxidase (HRP) for $20 \mathrm{~min}$. All incubation steps were performed at room temperature and between each step the plates were washed with Wash Buffer (code 895003; R\&D) to remove unbound molecules. After the last wash, a substrate solution prepared by mixing equal volumes of TMB (code 895001; $\mathrm{R} \& D$ ) and $\mathrm{H}_{2} \mathrm{O}_{2}$ (code 895000; $\mathrm{R} \& \mathrm{D}$ ) was added and the plates were incubated for 20 min. Following color development, sulfuric acid (code 895926; R\&D) was added to stop the reaction. The optical density was determined using a FLUOstar OPTIMA microplate reader from BMG Labtech, set to $450 \mathrm{~nm}$. To correct for optical imperfections in the plate a second reading was performed at $570 \mathrm{~nm}$ and readings were subtracted from the readings at $450 \mathrm{~nm}$. The data were linearized by plotting the log of the mean absorbance against the log of the concentration using Microsoft Excel version 1904 for Windows (Microsoft Corporation, Redmond, WA, USA). Serum CTLA-4, TNF- $\alpha$ and IL- 6 concentrations were determined using the curve fit equation $(y=m x+$ c) generated. The correlation coefficient between the fitted data and the actual data was greater than 0.99 for all assays.

Immunohistochemical (IHC) staining. The paraffin-embedded tumor tissue blocks were cut into $3 \mu \mathrm{m}$ thick sections and transferred to glass slides (SuperFrost Plus, Menzel-Gläser), dried at room temperature, baked in a heated chamber for $1 \mathrm{~h}$ at $64^{\circ} \mathrm{C}$, and then left overnight at room temperature. Deparaffinization and antigen retrieval were performed in a PT Link (Dako Denmark A/S) at $97^{\circ} \mathrm{C}$ for $20 \mathrm{~min}$, with low pH EnVision FLEX target retrieval solution (code DM829; Dako). Immunohistochemical staining was performed using the Novolink Max Polymer Detection System (Leica Biosystems). The samples were consecutively treated with: peroxidase block (code RE7157) for $20 \mathrm{~min}$; protein block (code RE7158) for $10 \mathrm{~min}$; the optimally diluted primary antibodies for $30 \mathrm{~min}$ (Table 4); the post-primary reagent (code RE7159) for $30 \mathrm{~min}$; and finally with the Novolink polymer (code RE7161) for $30 \mathrm{~min}$. The slides were washed between all incubation steps $2 \times 5 \mathrm{~min}$ in phosphate buffered saline $\mathrm{pH} 7.4$ (PBS). Afterwards, sections were stained with DAB chromogen (code 7162) for 5 min, and nuclei were counterstained with hematoxylin. Slides were dehydrated in an ethanol gradient, mounted with Entellan mounting medium and stored for later observation. Sections of feline lymph node specimens with confirmed high expression of the target molecules served as positive control. Sections of healthy mammary tissue were used as negative control.

Interpretation of IHC staining. Two of the authors (AU and JC) evaluated the scores for immunohistochemistry (IHC) grading and diagnostic accuracy. For CTLA-4 IHC staining evaluation, the results were recorded based on the intensity of the staining reaction on the cytoplasm, as well as the estimated percentage of positive interstitial lymphocytes. Interstitial lymphocytes were identified by morphology under a microscope after staining. The scores of percentages of positive cells were recorded as: $0(<1 \%), 1(1-5 \%), 2(6-30 \%)$ or $3(>30 \%)$. The scores of intensities of positive cells were recorded as: 0 (negative), $1+$ (weak), $2+$ (moderate), or $3+$ (strong) (Fig. 3). The percentage of positive cells and intensity scores were then multiplied to obtain a final IHC score ${ }^{32}$. The FoxP3 expression in interstitial lymphocytes was evaluated in a similar way.

Statistical analysis. Statistical analysis was carried out using GraphPad Prism version 8.11 for Windows (GraphPad Software, La Jolla, CA, USA). The values $\mathrm{p}<0.05(*), \mathrm{p}<0.01(* *)$ and $\mathrm{p}<0.001(* * *)$ were considered statistically significant. Differences between serum CTLA- 4 and cytokine levels in healthy and diseased animals were assessed using the Mann-Whitney $U$ test. The association between serum CTLA- 4 levels and various clinicopathological criteria were analyzed by the Kruskal-Wallis test. To control the family wise error rate we adjusted the significance level as $\alpha=\alpha / m$ where $m$ is the number of simultaneously tested hypotheses ${ }^{33}$. Pearson correlation was used to assess correlations between CTLA- 4 and TNF- $\alpha /$ IL- 6 serum levels. Cats were divided into positive- and negative-expression groups for lymphocyte CTLA- 4 and FoxP3 expression, IHC scores of 0 and 1 were defined as negative and scores $>1$ as positive. These definitions accounted for the median scores and minimized the difference between the number of animals classified as negative and those classified as positive. Correlations between CTLA- 4 serum levels and lymphocyte CTLA-4 and FoxP3 expressions were assessed using the Spearman rank and Mann-Whitney U tests.

Received: 27 September 2019; Accepted: 22 January 2020;

Published online: 02 March 2020

\section{References}

1. Cannon, C. Cats, Cancer and Comparative Oncology. Vet. Sci. 2, 111-126 (2015).

2. Thomas, R. Cytogenomics of Feline Cancers: Advances and Opportunities. Vet. Sci. 2, 246-258 (2015).

3. De Vico, G. \& Maiolino, P. Canine and Feline Models for Cancer. In Sourcebook of Models for Biomedical Research 677-682 (Humana Press, 2008). https://doi.org/10.1007/978-1-59745-285-4_70. 
4. De Maria, R. et al. Spontaneous feline mammary carcinoma is a model of HER2 overexpressing poor prognosis human breast cancer. Cancer Res. 65, 907-912 (2005).

5. Soares, M., Correia, J., Peleteiro, M. C. \& Ferreira, F. St Gallen molecular subtypes in feline mammary carcinoma and paired metastases-disease progression and clinical implications from a 3-year follow-up study. Tumor Biol. 37, 4053-4064 (2016).

6. Soares, M. et al. Serum HER2 levels are increased in cats with mammary carcinomas and predict tissue HER2 status. Oncotarget 7, 17314-17326 (2016).

7. Ben-Baruch, A. Host microenvironment in breast cancer development: inflammatory cells, cytokines and chemokines in breast cancer progression: reciprocal tumor-microenvironment interactions. Breast Cancer Res. 5, 31-6 (2003).

8. Sharpe, A. H. \& Freeman, G. J. The B7-CD28 superfamily. Nat. Rev. Immunol. 2, 116-126 (2002)

9. Ohno, K., Fujiki, M., Khatlani, T. S., Inokuma, H. \& Onishi, T. Cloning of Feline cDNA Encoding the Cytotoxic T LymphocyteAssociated Antigen 4. J. Vet. Med. Sci. 61, 1241-1244 (1999).

10. Oaks, M. K. et al. A native soluble form of CTLA-4. Cell. Immunol. 201, 144-153 (2000).

11. Zhang, X.-F. et al. Cytotoxic T lymphocyte antigen-4 expression in esophageal carcinoma: implications for prognosis. Oncotarget 7, 26670-26679 (2016).

12. Liu, Q. et al. The prognostic value of cytotoxic T-lymphocyte antigen 4 in cancers: a systematic review and meta-analysis. Sci. Rep. 7, 1-10 (2017).

13. Erfani, N., Razmkhah, M. \& Ghaderi, A. Circulating soluble CTLA4 (sCTLA4) is elevated in patients with breast cancer. Cancer Invest. 28, 828-832 (2010).

14. Wing, K. et al. CTLA-4 Control over Foxp3+ Regulatory T Cell Function. Science (80-.). 322(5899), 271-275 (2008).

15. Bou-Dargham, M. J., Liu, Y., Sang, Q.-X. A. \& Zhang, J. Subgrouping breast cancer patients based on immune evasion mechanisms unravels a high involvement of transforming growth factor-beta and decoy receptor 3. PLoS One 13, e0207799 (2018).

16. Lu, L., Bai, Y. \& Wang, Z. Elevated T cell activation score is associated with improved survival of breast cancer. Breast Cancer Res. Treat. 164, 689-696 (2017).

17. Liu, Q. et al. Soluble cytotoxic T-lymphocyte antigen 4: A favorable predictor in malignant tumors after therapy. Onco. Targets. Ther. 10, 2147-2154 (2017).

18. Simone, R. et al. The soluble form of CTLA-4 from serum of patients with autoimmune diseases regulates T-cell responses. Biomed Res. Int. 2014, 215763 (2014).

19. Sparger, E. E. et al. Investigation of immune cell markers in feline oral squamous cell carcinoma. Vet. Immunol. Immunopathol. 202, 52-62 (2018).

20. Ward, F. J. et al. The soluble isoform of CTLA-4 as a regulator of T-cell responses. Eur. J. Immunol. 43, 1274-1285 (2013).

21. Sakthivel, P. et al. Circulating soluble CTLA-4 is related to inflammatory markers in the 70 year old population. Scand. J. Clin. Lab. Invest. 70, 237-243 (2010)

22. Saverino, D. et al. Soluble CTLA-4 in autoimmune thyroid diseases: Relationship with clinical status and possible role in the immune response dysregulation. Clin. Immunol. 123, 190-198 (2007).

23. Pérez-García, A. et al. Kinetics of the CTLA-4 isoforms expression after T-lymphocyte activation and role of the promoter polymorphisms on CTLA-4 gene transcription. Hum. Immunol. 74, 1219-1224 (2013).

24. Salvi, S. et al. Evaluation of CTLA-4 expression and relevance as a novel prognostic factor in patients with non-small cell lung cancer. Cancer Immunol. Immunother. 61, 1463-1472 (2012)

25. Santos, S. et al. ERBB2 in Cat Mammary Neoplasias Disclosed a Positive Correlation between RNA and Protein Low Expression Levels: A Model for erbB-2 Negative Human Breast Cancer. PLoS One 8, e83673 (2013).

26. Persson, J. et al. Intratumoral Expression of CTLA4 Monoclonal Antibody Induces Immunosuppressive NKT Cells in a Mouse Model of Breast Cancer with Tolerance to Her2/neu. Mol. Ther. 19, S88-S89 (2011).

27. E. Goldberg, J. \& L. Schwertfeger, K. Proinflammatory Cytokines in Breast Cancer: Mechanisms of Action and Potential Targets for Therapeutics. Curr. Drug Targets 11, 1133-1146 (2010).

28. Grohmann, U. et al. CTLA-4-Ig regulates tryptophan catabolism in vivo. Nat. Immunol. 3, 1097-1101 (2002).

29. Lee, S., Cho, E. Y., Park, Y. H., Ahn, J. S. \& Im, Y.-H. Prognostic impact of FOXP3 expression in triple-negative breast cancer. Acta Oncol. (Madr). 52, 73-81 (2013).

30. Poschke, I., De Boniface, J., Mao, Y. \& Kiessling, R. Tumor-induced changes in the phenotype of blood-derived and tumor-associated T cells of early stage breast cancer patients. Int. J. Cancer 131, 1611-1620 (2012).

31. Jago, C. B., Yates, J., Olsen Saraiva Câmara, N., Lechler, R. I. \& Lombardi, G. Differential expression of CTLA-4 among T cell subsets. Clin. Exp. Immunol. 136, 463-471 (2004).

32. Lan, G. et al. Cytotoxic T lymphocyte associated antigen 4 expression predicts poor prognosis in luminal B HER2-negative breast cancer. Oncol. Lett. 15, 5093-5097 (2018).

33. Chen, S.-Y., Feng, Z. \& Yi, X. A general introduction to adjustment for multiple comparisons. J. Thorac. Dis. 9, 1725-1729 (2017).

\section{Acknowledgements}

This work was supported by Fundação para a Ciência e a Tecnologia (Portugal) through the projects PTDC/ CVTEPI/3638/2014 and CIISA-UIDP/CVT/00276/2020. C.N. is receipt of a PhD fellowship from University of Lisbon. The authors would like to thank Dra. Andreia Gameiro (FMV/ULisboa) for her technical support on the preparation of tissue section for immunohistochemistry.

\section{Author contributions}

A.U. and F.F. designed the experiments and the conception of the study. A.U., C.N., M.J.S. and J.C. carried out the experiments. A.U. and F.F. analyzed the data. A.U and F.F. drafted and reviewed the manuscript.

\section{Competing interests}

The authors declare no competing interests.

\section{Additional information}

Correspondence and requests for materials should be addressed to F.F.

Reprints and permissions information is available at www.nature.com/reprints.

Publisher's note Springer Nature remains neutral with regard to jurisdictional claims in published maps and institutional affiliations. 
(c) (i) Open Access This article is licensed under a Creative Commons Attribution 4.0 International License, which permits use, sharing, adaptation, distribution and reproduction in any medium or format, as long as you give appropriate credit to the original author(s) and the source, provide a link to the Creative Commons license, and indicate if changes were made. The images or other third party material in this article are included in the article's Creative Commons license, unless indicated otherwise in a credit line to the material. If material is not included in the article's Creative Commons license and your intended use is not permitted by statutory regulation or exceeds the permitted use, you will need to obtain permission directly from the copyright holder. To view a copy of this license, visit http://creativecommons.org/licenses/by/4.0/.

(C) The Author(s) 2020 